\title{
The Effects of Sugars and Ethylene on Apospory and Regeneration in Ceratopteris richardii
}

\author{
Linh T. Bui, Amelia Hurst, Erin E. Irish, Chi-Lien Cheng*
}

Department of Biology, The University of Iowa, Iowa City, USA.

Email: *chi-lien-cheng@uiowa.edu

Received May $5^{\text {th }}, 2012$; revised June $2^{\text {nd }}, 2012$; accepted June $10^{\text {th }}, 2012$

\begin{abstract}
In land plants, two distinct generations, gametophyte and sporophyte, alternate to complete the life cycle. Sporophytes undergo meiosis to produce spores, from which gametophytes develop. Gametophytes produce gametes, which participate in fertilization to produce the zygote, the first cell of the sporophyte generation. In addition to this sexual reproduction pathway, some fern species can undergo apospory or apogamy, processes that bypass meiosis or fertilization, respectively, to alternate between the two generations without changing the chromosome number. Apospory is inducible in the laboratory in various fern species simply by altering the sugar level in the media. In sporophytes induced to undergo apospory, sporophyte regeneration is also observed. The ratio of aposporous gametophytes to regenerated sporophytes varies, in a manner consistent with being dependent on sugar level. Whereas the sugar signaling pathway is yet to be elucidated in lower plants, in angiosperms it has been shown to play a regulatory role in controlling essential processes including flowering and embryo development, which give rise to the gametophyte and the next sporophyte generation, respectively. Here, we present evidence for the role of different sugar levels on the balance of apospory and regeneration in the fern Ceratopteris richardii. The demonstration of crosstalk between sugar signaling and the hormone ethylene signaling in angiosperms prompted us to test the effects of this hormone in combination with sugar on apospory vs. regeneration. These results provide insight into how a group of redifferentiating cells determines which generation to become and lay the groundwork for further analysis of this asexual pathway.
\end{abstract}

Keywords: Ceratopteris; Fern; Apospory; Regeneration; Sugar; Ethylene; Gametophyte; Sporophyte

\section{Introduction}

In all land plants, the life cycle is characterized by an alternation between two generations; each develops into a multicellular body. Together the two generations, the haploid gametophyte and the diploid sporophyte, undergo the core events of sexual reproduction to complete the life cycle. Sporophytes produce haploid spores by specialized cells undergoing meiosis; these spores develop into multicellular gametophytes which produce gametes. The fusion of male and female gametes during fertilization produces the zygote, the first cell of the sporophyte generation, as well as restoring ploidy levels. In angiosperms, spores and resulting gametophytes are ephemeral, embedded deep in the flower, and dependent on the sporophyte. In ferns, mature spores are shed from the sporophytes into the environment where, upon germination, they develop into photosynthetic gametophytes, from which gametes are produced. Thus, in contrast to angiosperms, both generations of ferns are free-living

${ }^{*}$ Corresponding author. entities.

In addition to sexual reproduction pathway via meiosis and fertilization, some fern species can reproduce asexually through apogamy or apospory. In nature, many fern species are obligately apogamous: gametophytes lack functional archegonia or antheridia and sporophytes arise directly from the gametophyte asexually $[1,2]$. During sporogenesis, a compensatory mechanism with formation of restitution nuclei acts to give rise to diplospores with the same chromosome numbers as the apogamous sporophytes [3]. Obligate apospory also occurs in ferns [4], albeit rarely [3]. In apospory, diploid gametophytes are produced from somatic cells of sporophytes in the absence of meiosis. Both processes can be induced in the laboratory in the homosporous fern Ceratopteris richardii simply by altering the level of sugar supplement in the growth media, and in the case of apogamy, by also preventing fertilization [5]. Glucose at $2.5 \%(\mathrm{w} / \mathrm{v})$ was found to be optimal for the induction of apogamy [5] and medium with no sugar or $0.01 \%(\mathrm{w} / \mathrm{v})$ glucose supplement was used in apospory [6-8] in C. richardii. Neither 
apogamy nor apospory, even under the optimum conditions, is exclusively so; gametophytes and sporophytes regenerate in large numbers during apogamy and apospory, respectively (Cordle and Bui, unpublished results). This observation is well known and in many species, both gametophytes and young sporophytes have been used in regenerative propagation (for a recent review, see [9]).

All sugars found in plants ultimately come from photosynthesis. Many are essential structural and metabolic molecules supporting plant growth and development. Beyond these functions, hexose and some disaccharides have been shown to participate in signaling pathways in vascular plants leading to processes such as seed germination, seedling growth, leaf morphogenesis, embryo development, flowering and senescence (for reviews, see $[10,11])$. Much of the hexose signaling in vascular plants has been through specific members of the hexokinases $[11,12]$. In the seedless plants Physcomitrella and Ceratopteris, many more hexokinase genes exist and their roles in signaling have yet to be defined ([13]; Cheng, unpublished results); to date, a stromal HXK was shown to be responsible for glucose-mediated growth of caulonema of the Physcomitrella gametophytes [14]. Although sucrose can be converted to hexose in the plant, it may also act as a signal itself: for example, in Vicia faba, sucrose accumulation correlates with the switch from cell division to differentiation during embryo development [15].

The discovery that 1-aminocyclopropane-1-carboxylic acid (ACC) is able to counteract the inhibition of seedling greening in Arabidopsis caused by high concentration of exogenous glucose prompted the investigation of a possible crosstalk between ethylene and sugar signaling $[16,17]$. Examination of mutants defective in or hypersensitive to glucose and ethylene signaling further supports the occurrence of crosstalk [18-20]. In general, ethylene antagonizes the effect of sugar responses; however, the crosstalk between glucose and ethylene signaling depends on the glucose level applied and the developmental stage of the plant [21]. In ferns, ethylene has been reported to promote the elongation of dark-grown filaments and to inhibit rhizoid growth in gametophytes [22], and regeneration of sporophytes [23]. The major pathway of ethylene biosynthesis in some fern species, however, is not through methionine and ACC but by a yet unidentified pathway [24,25]. Nonetheless, ethylene is produced in seed and seedless plants alike [26]. To study the effect of ethylene on developmental processes in seedless plants, researchers have used ethephon (2-chloroethylphosphonic acid) and silver thiosulfate (STS) to increase ethylene level or block ethylene action, respectively [23, 27]. Ethephon degrades to yield ethylene in an alkaline solution, and has been shown to be effective in causing responses unique for ethylene treatment when applied to plants and in tissue culture [28]. On the other hand, STS is an inhibitor that blocks ethylene action. Silver ions in STS replace the copper or zinc of the ethylene receptors and hence prevent them from responding to ethylene, exogenous or endogenous [29].

Here, we used the model fern C. richardii to investigate the effects of different sugar concentrations on the production of aposporous gametophytes and on sporophyte regeneration from excised sporophyte leaves. The demonstration of crosstalk between sugar signaling and the hormone ethylene signaling in angiosperms prompted us to test the effects of this hormone in combination with low levels of sugars on apospory and on regeneration.

\section{Materials and Methods}

\subsection{Plant Material and Growth Condition}

C. richardii plants used in these experiments were of the wild-type genotypes Rn3 or Hnn (Carolina Biological Supply, Burlington, NC). Spore germination and gametophyte culture conditions were as described by Hickok et al. (1995) [30] and Cordle et al. (2007) [5]. Spores were inoculated at a density of 150 - 300 spores per plate containing a basal medium (BM) which is halfstrength Murashige and Skoog salts (MS) at pH 6.0 with $0.8 \%$ agarose. Gametophyte culture plates were maintained at $28^{\circ} \mathrm{C}$ in humidity domes under 16-h light/8-h dark cycle. Light was provided by Philips Agro-Lite fluorescent bulbs (Philips Lightning Company, Somerset, $\mathrm{NJ})$ at $90-100 \mu \mathrm{M} \cdot \mathrm{m}^{-2} \cdot \mathrm{s}^{-1} \cdot 14$ days after germination, water was added to the gametophyte culture plates to initiate fertilization. 7 days after fertilization, young sporophyte leaves (leaf one after fertilization) were used for apospory induction experiments.

\subsection{Apospory Induction Experiment}

Young sporophyte leaves were excised from the gametophytes and placed on an apospory-inductive medium [BM supplemented with $0.5 \%$ (w/v) sugar, either sucrose or glucose]. Plates were sealed with micropore tape (3 M Health Care, MN, USA) and put inside a humidity dome. The same light conditions used for spore germination and gametophyte growth was used here. The number of explants having either aposporous gametophytes or regenerative sporophytes or both was counted at different time points from week 2 to week 7 using a dissecting microscope (Leica Wild MC3). Aposporous gametophytes are distinguished from regenerative sporophytes by the difference in thickness of the thalli, as gametophytes are single-cell-layered and sporophytes are multilayered, as well as the appearance of archegonia and antheridia, gametophyte-specific organs. Frequency of apospory was 
calculated based on the number of explants having aposporous gametophytes divided by the total number of explants on the plates multiplied by 100 . Frequency of regeneration was calculated based on the number of explants having regenerative sporophytes divided by the total number of explants on the plates multiplied by 100 . Explants that had both aposporous gametophytes and regenerative sporophytes were counted in both calculations. The total numbers of aposporous gametophytes and regenerative sporophytes were counted at the end of each experiment (day 56 after plating on apospory-inductive medium).

\subsection{Histology}

Images of aposporous gametophytes and regenerative sporophytes were taken using a dissecting microscope and a Dino-Lite digital camera. Five explants having aposporous gametophytes were chosen to observe gametophyte structures by scanning electron microscopy (SEM) in the University of Iowa Central Microscopy Research Facility.

For ploidy level determination using Hoechst dye, at least five aposporous gametophytes, young sporophytes (diploid standard), and spore-derived gametophytes (haploid standard) were used. Sample treatment was as described in detail in Cordle et al., 2007 [5]. Briefly, each cohort of samples were cleared in $95 \%$ ethanol overnight at $4{ }^{\circ} \mathrm{C}$, rinsed in water and immersed in 40 $\mu \mathrm{g} \cdot \mathrm{mL}^{-1}$ Hoechst 33342 dye for $2.5 \mathrm{~h}$, rinsed twice in distilled water, mounted on slides with glycerol, and imaged under DAPI filtered UV light. Fluorescence intensity was quantified using ImageJ $1.37 \mathrm{j}$ software (http:// rsb.info.nih.gov/ij/). The images were converted to 8-bit gray scale and binarized using the intensity thresholding function. The selected fluorescing nuclei were regions of interest. Mean gray values were then obtained from the defined regions of interest and converted into relative intensities. Mean pixel intensities of 7 to 18 in-focus nuclei from each putatively apogamous, haploid standard, and diploid standard were compared to quantify ploidy of the corresponding tissues (Cordle et al., 2007) [5].

\subsection{Ethylene Experiments}

Ethephon (2-chloroethylphosphonic acid, Sigma) was added to the media after filter sterilization. STS solution was prepared according to manufacturer's instructions (PhytoTechnology Laboratories, Inc.) Briefly, a $0.02 \mathrm{M}$ STS solution was prepared by slowly pouring $20 \mathrm{~mL}$ of $0.1 \mathrm{M}$ silver nitrate stock solution into $80 \mathrm{~mL}$ of $0.1 \mathrm{M}$ STS stock solution prior to use and then filter sterilized. Numbers of sporophyte leaves bearing at least one aposporous gametophyte and those with regenerative sporophytes were determined weekly. The total number of gametophytes and sporophytes were tallied at day 56 and the ratios calculated as described in the section Apospory Induction Experiment.

\subsection{Statistical Analysis}

The JMP9 program (SAS Institute Inc.), specifically oneway ANOVA and T-student test, was applied to ascertain the significant differences between the total numbers of aposporous gametophytes and regenerative sporophytes in different media at the end of each experiment. Graphs were plotted using RStudio (RStudio, Inc.) and Excel programs.

\section{Results}

\subsection{Apospory and Regeneration Can Occur on the Same Explants}

The first leaf of $7 d$ old sporophytes, when placed on inductive medium, regenerated sporophytes (Figures 1(a), (c), (e)) and also produced aposporous gametophytes (Figures 1(a)-(c)). Sugar supplemented at low concentration $(0.5 \%$ glucose) promoted apospory (Figure 1(a)) and at high concentration $(2.5 \%$ glucose $)$ promoted regeneration (Figure 1(e)). The majority of the gametophytes formed on low concentration of glucose had normal cordate morphology (Figure 1(b)) in contrast to the abnormal, elongated morphology when formed on high concentration of glucose (Figure 1(d)). Both cordate and elongated gametophytes developed archegonia and antheridia (Figures 1(f), (g)). The antheridia produced sperm (Figure 1(k)) and archegonia produced eggs that formed zygotes that developed into sporophytes after fertilization (data not shown). Shoot morphology of the regenerative sporophytes were similar on high and low concentrations of glucose (data not shown) but root growth was more pronounced in high concentration of glucose (Figure 1(e)). Results shown here were conducted with strain Rn3. When strain Hnn was used, there was no apparent difference in efficiency of regeneration and apospory and their respective morphology (data not shown).

Unlike spore-derived gametophytes, which are haploid (Figure 1(h)), having been produced without meiosis, aposporous gametophytes were diploid (Figure 1(i)) having fluorescence intensity (Figure 2) similar to that of zygote-derived sporophytes (Figure 1(j)).

\subsection{Sugar Levels Affect Regeneration and Apospory in C. richardii}

Our observation that both regeneration and apospory often occurred on the same explant (Figure 1(c)) and that the likelihood of one over the other was affected by the levels of glucose (Figures 1(a), (e)) prompted us to carry out a time-course study of regeneration and apospory on 


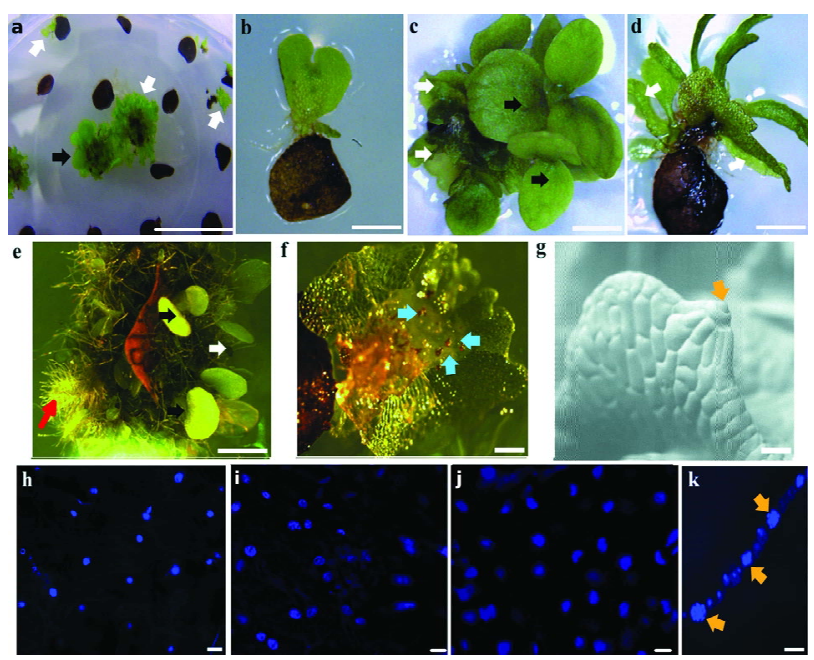

Figure 1. Apospory and regeneration from sporophyte leaves. Explants grown on BM supplemented with $0.5 \%$ glucose (a), (b), (f) or with $2.5 \%$ glucose (c), (d), (e) for $56 \mathrm{~d}$ (a), (c), (d) or for $49 \mathrm{~d}$ (b), (e) showing aposporous gametophytes and regenerative sporophytes from explants (a), an aposporous gametophyte attaching to a nearly dead sporophyte explant (b), regenerative sporophytes and aposporous gametophytes arising from the same explants (c), elongated aposporous gametophytes (d), regenerative sporophytes with proliferating roots (e), and aposporous gametophytes with well developed archegonia (f) and antheridia (g). Hoechst DNA dye intensity was used as a ploidy marker by comparing nuclear fluorescence of spore derived gametophytes (h), aposporous gametophytes (i) and zygote derived sporophyte tissues (j), and Hoechst DNA dye staining of the antheridia containing sperm on the edge of an aposporous gametophyte (k). White, black, and red arrows indicate aposporous gametophytes, regenerative sporophytes, and proliferating roots, respectively; blue and orange arrows indicate archegonia and antheridia, respectively. Bar $=1$ $\mathrm{mm}(\mathrm{a})-(\mathrm{g})$, bar $=5 \mu \mathrm{m}(\mathrm{h})-(\mathrm{j})$, bar $=0.05 \mathrm{~mm}(\mathrm{k})$.

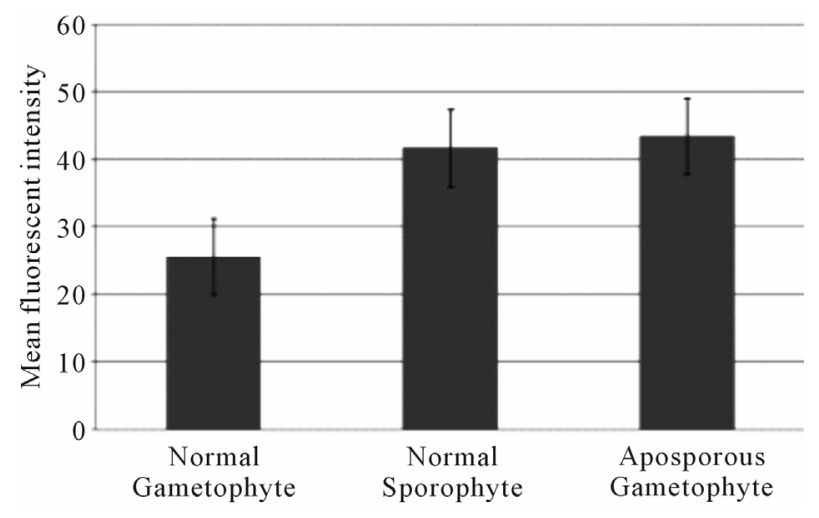

Figure 2. Quantitative determination of ploidy levels by Hoechst dye intensity. Using Image $J$, the mean pixel intensity was calculated from at least 21 in-focus nuclei from each of the aposporous gametophytes, normal (spore-derived) gametophytes, and normal (zygote-derived) sporophytes, with the highest mean value set at 100 . Bars represent the relative intensity. Vertical lines represent standard deviation. high $(2.5 \%)$ and low $(0.5 \%)$ levels of glucose and sucrose. After explants were placed on media, the number of gametophytes and sporophytes was counted weekly. No signs of regeneration or apospory could be observed in week 1 , but both could be detected and distinguished starting at week 2. At that time, the majority of the explants $(45 \%-55 \%$ on low concentration of sugars and $70 \%-80 \%$ on high concentration of sugars) had already given rise to at least one detectable sporophyte when compared with the maximum number of explants bearing sporophytes at week 5 ( $85 \%$ of the explants on high sugar concentration; $55 \%$ and $72 \%$ on low concentration of glucose and sucrose, respectively) (Figure 3(a)). In contrast, apospory proceeded more slowly than regeneration; in week 2, gametophytes were detected on fewer than half of the explants $(20 \%$ on low concentration of sugars and $10 \%$ on high concentration of sugars) when compared with the maximum number of explants showing apospory at week $5(40 \%$ of the explants on low concentration of sugars; $27 \%$ on high concentration of sugars) (Figure 3(b)).

Compared with basal medium without supplemented sugar, low sugar level increased both regeneration and apospory but favored apospory. At high levels, sugar had differential effects on apospory and regeneration, promoting regeneration but inhibiting apospory. At day 56, the ratios of total gametophyte number/sporophyte number on low concentration of sugars are much higher than those on high concentration of sugars $(p<0.1)$ (Figure 3(c)). Under all growth conditions, regeneration occurred more efficiently than did apospory. Not only did more explants bear sporophytes than bear gametophytes (Figures 3(a), (b)), but individual explants also bore more sporophytes (average 5.6 per explant) than gametophytes (average 2.5) (data not shown; Figure 3(c)). The two sugars tested showed no significant difference on apospory and regeneration except that sucrose at 2.5\% seemed to be more effective than glucose at $2.5 \%$ in promoting regeneration after week 4 (Figures 3(a), (c)).

\subsection{Ethylene Plays an Inductive Role on Apospory and Regeneration in C. richardii}

Ethylene has been shown to accumulate in wounded tissues in tissue culture [29] and is involved in various plant developmental pathways, including female gametophyte development [31]. Therefore, we conducted the following experiments to examine the role of ethylene in apospory and regeneration in C. richardii. Two different chemicals, ethephon and STS, were added to $0.5 \%$ glucose medium, the concentration optimal for apospory induction (Figure 3), either to increase the ethylene concentration inside the plant cells or to block ethylene action, respectively. Both ethephon and STS have been 


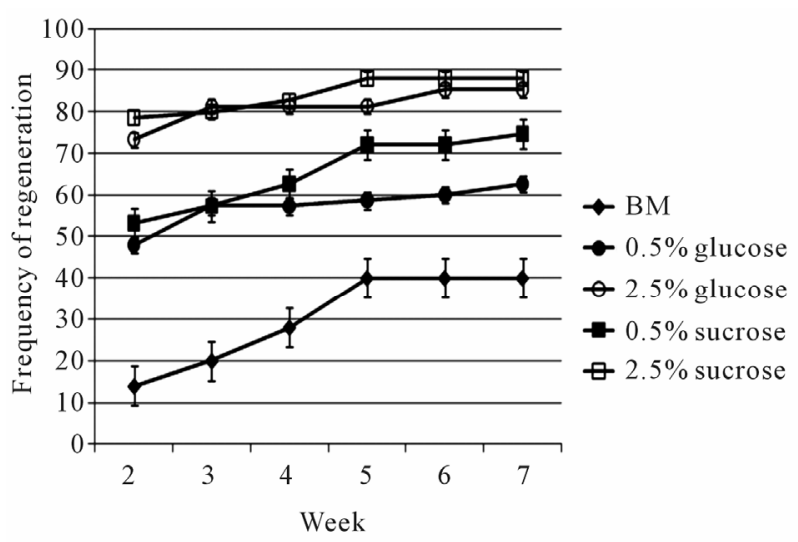

(a)

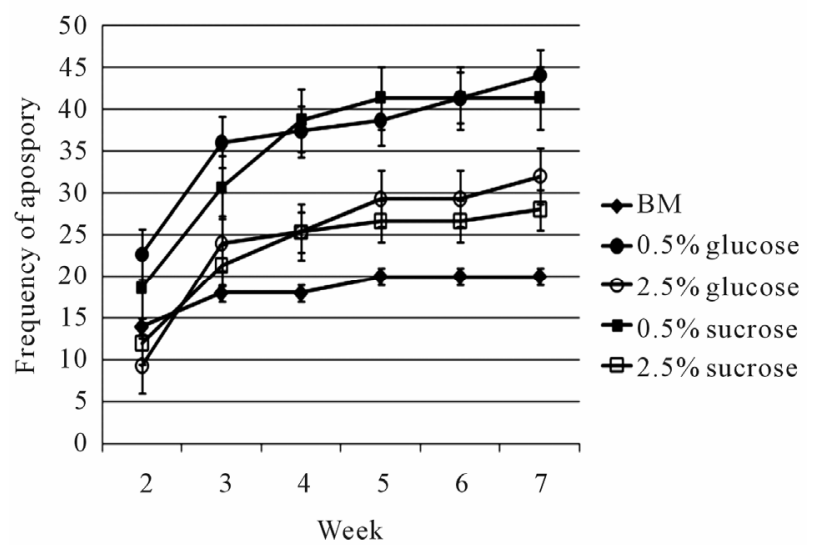

(b)

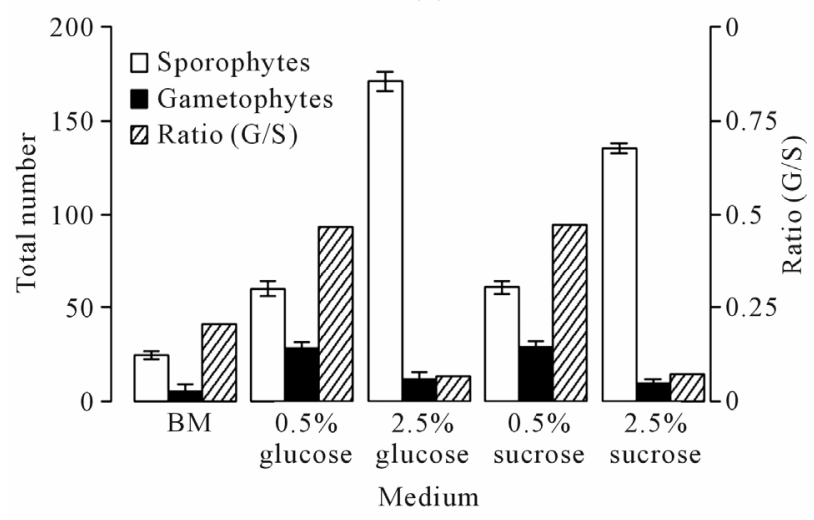

(c)

Figure 3. Effects of sugar concentration on apospory and regeneration. Frequency of regeneration is defined as percentage of explants among total explants plated on each medium that have produced 1 or more sporophyte at any time point; these explants may have any number of gametophytes (a). Frequency of apospory is defined as percentage of explants among total explants plated on each medium that have produced 1 or more gametophyte at any time point; these explants may have any number of sporophytes (b). At the end of the experiment (d 56), total number of sporophytes and total number of gametophytes on all the explants were counted (c). Means (light and medium gray bars) are averages from three different experiments: lines represent standard errors. used widely to study ethylene effects in lower plants with little or no toxic effects on plant tissues reported [7,23].

There was no sign of apospory or regeneration observed in any kind of media in week 1 . In week 2 , regeneration occurred in all media tested, ranging from $3 \%$ (100 mg/L ethephon) to $23 \%(0 \mathrm{mg} / \mathrm{L}$ ethephon) (Figure 4(a)). However, apospory did not occur on any explants until week 3 in most media tested. In week $3,10 \%$ of the explants on media with $20 \mathrm{mg} / \mathrm{L}$ ethephon showed aposporous gametophytes, the highest percentage of all the media combination (Figure 4(b)). From week 4 to week 8 , more detectable gametophytes were observed on all media, with the highest number observed on medium with $60 \mathrm{mg} / \mathrm{L}$ ethephon (Figure 4(b)). The total number of gametophytes at day 56 (end of the experiment) was also highest on this medium with an average of 45 aposporous gametophytes per plate (Figure 4(c)). Ethephon also induces regeneration since the percentage of regeneration was significantly different among all the media tested, with $20 \mathrm{mg} / \mathrm{L}$ ethephon resulted in the highest amount of detectable sporophytes (Figures 4(b), (c)). However, a high ethephon concentration $(100 \mathrm{mg} / \mathrm{L})$ inhibited both apospory (Figures 4(a), (c)) and regeneration (Figures 4(b), (c)).

To further confirm the promoting role of ethylene in both apospory and regeneration, we used STS to block ethylene action. As in the ethephon experiment, neither apospory nor regeneration could be observed during the first week of this experiment. Regeneration occurred at week 2 with the appearance of newly formed sporophytes on most of the media. There was a decreasing number of explants showed regeneration with the increasing concentration of STS; at $80 \mu \mathrm{M}$ STS only $2 \%$ of the explants showed any regeneration compared to $35 \%$ of the explants showing regeneration as early as week 5 when grown on medium without STS (Figure 5(a)). Similarly, STS inhibited apospory in a dosage-dependent manner; however, the effect is more potent: at $20 \mu \mathrm{M}$ STS, apospory is completely inhibited until week 8 (Figure 5(b)). Interestingly, $5 \mu \mathrm{M}$ STS in the medium seemed to hasten apospory initially (at week 3 ) compared with no STS treatment.

\section{Discussion}

Here we have provided evidence for the effects of sugars and ethylene on both apospory and regeneration in the fern Ceratopteris richardii. The aposporous gametophytes appear in many different forms, ranging from an intermediate between sporophyte and gametophyte to an elongated cordate shape to a normal form. All mature cordate aposporous gametophytes examined had all the hallmarks of spore-derived gametophytes such as single cell layer thickness, well-developed archegonia and antheridia, 


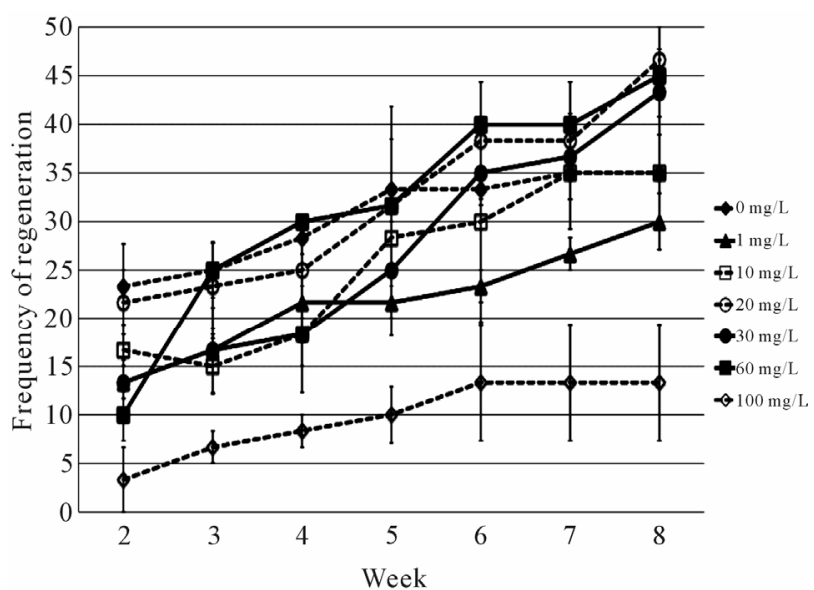

(a)

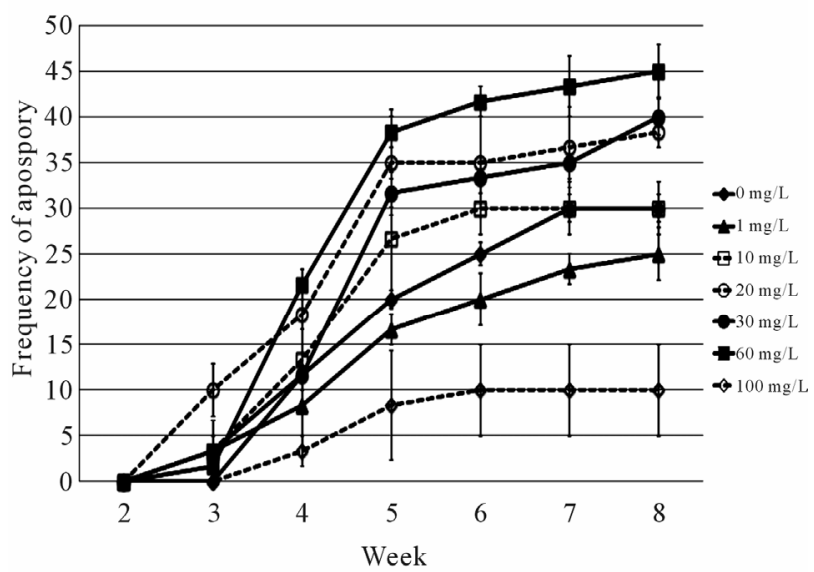

(b)

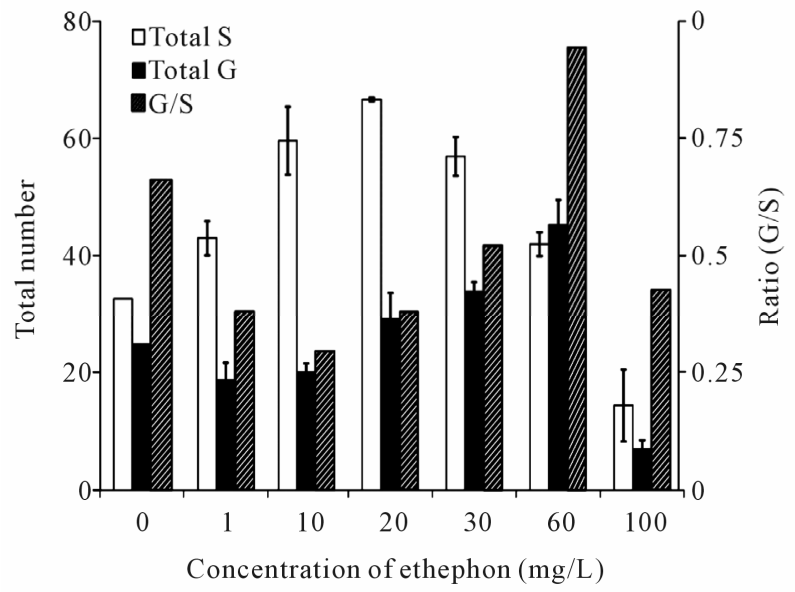

(c)

Figure 4. Effects of ethephon on apospory and regeneration. Explants were treated with increasing amounts of ethephon $(\mu M)$. Frequency of regeneration is defined as in Figure 3(a) (a). Frequency of apospory is defined as in Figure 3(b) (b). At the end of the experiment (d 56), all sporophytes and gametophytes were counted and frequency was calculated as described in Figure 3(c) (c). Means (light and medium gray bars) are averages from three different experiments: lines represent standard errors.

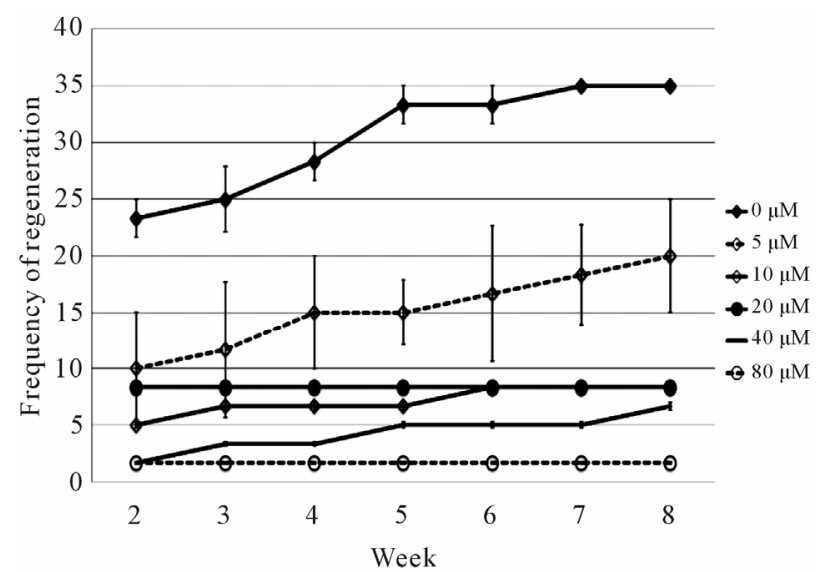

(a)

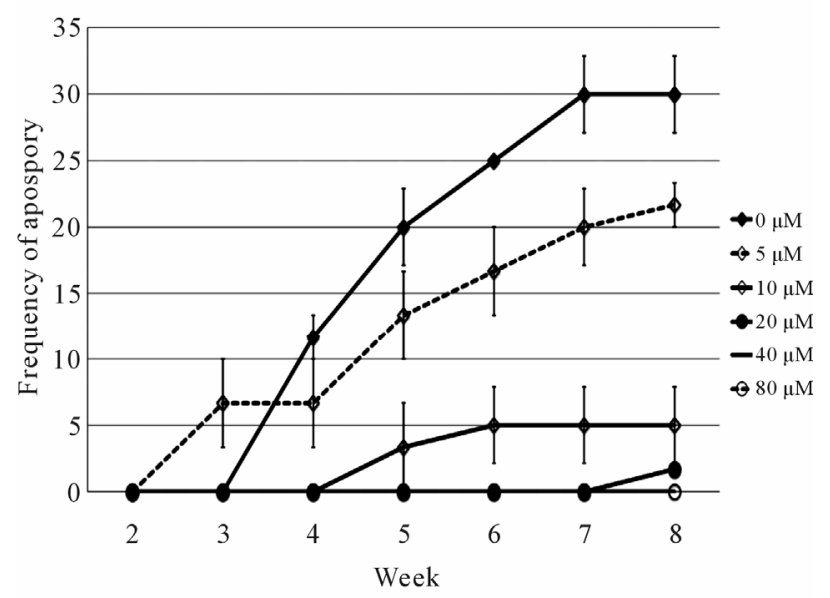

(b)

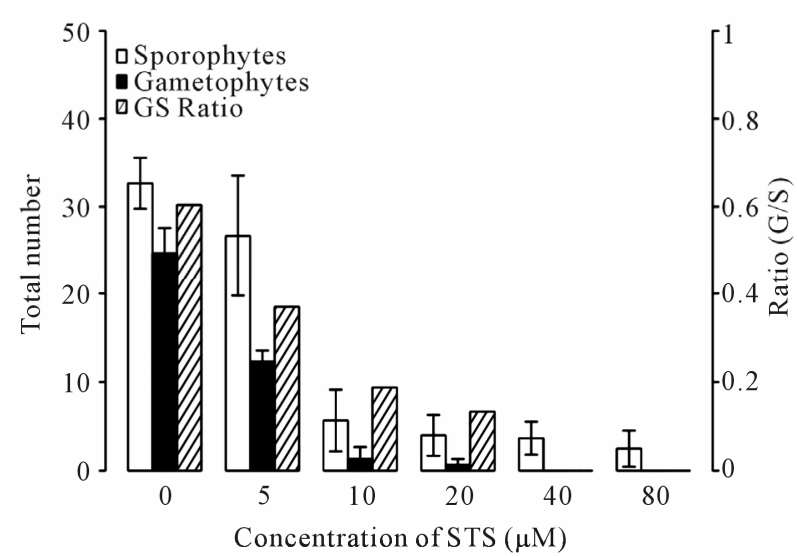

(c)

Figure 5. Effects of STS on apospory and regeneration. Explants were treated with increasing amounts of STS $(\mu M)$. Frequency of regeneration is defined as in Figure 3(a) (a). Frequency of apospory is defined as in Figure 3(b) (b). At the end of the experiment (d 56), total numbers of sporophytes and gametophytes were counted and calculated as described in Figure 3(c) (c). Means (light and medium gray bars) are averages from three different experiments; lines represent standard errors. 
and the lack of stomata. In fact, they are indistinguishable from the spore-derived, haploid gametophytes except may be slightly larger (data not shown). They are sexually competent: when fertilized, they form zygotederived sporophytes, which are presumably tetraploid. Relative DNA content when compared with haploid gametophytes and sporophyte tissues confirmed the diploid state of all aposporous gametophytes examined, indicating an alternation of generations in the absence of meiosis. Most regenerated sporophytes were identical to zygote-derived sporophytes and develop normally into mature ferns with functional roots and leaves (not shown).

Sugars have been shown to play a role as a signaling molecule in various processes during development of seed plants (for reviews, see [10,11]). Previous studies have shown that the concentration of sugar in the media is also an important factor in promoting or inhibiting the two asexual pathways, apogamy and apospory, in many fern species [5,7,8,32,33]. In C. richardii, high sugar level $(2.5 \%$ glucose $)$ promotes the formation of haploid apogamous sporophytes from gametophytes in the absence of fertilization [5] while low sugar level $(0.01 \%$ glucose) or no sugar in the medium promotes the formation of diploid aposporous gametophytes from sporophytes $[7,8]$. Here we showed that sugars (glucose and sucrose) at low (0.5\%) and high (2.5\%) levels have opposite effects in promoting regeneration and apospory in the fern $C$. richardii. High sugar levels promote regeneration but inhibit apospory while low sucrose or glucose at $0.5 \%$ favors apospory over regeneration. Note that high sugar levels favor sporophyte development, whether through regeneration from a sporophyte leaf or through apogamy, independent of the ploidy of the explant. That basal medium is probably as poor in promoting regeneration as in promoting apospory from lack of sufficient carbohydrates is suggested by the browning explants during long periods of culture on the agar plates.

The nutritional effect of sugar on both apospory and regeneration has been suspected because the differences between the two generations imply different nutrient requirement [32]. Gametophytes are small, simple and limited to moist environment while sporophytes are highly complex plants. In addition, on high sugar media with $2.5 \%$ sucrose or glucose, most explants turned to dark brown color and showed necrosis after six weeks in contrast to explants grown on $0.5 \%$ sucrose or glucose (data not shown), indicating that high levels of sugar hasten senescence. High sugar levels also have been shown to trigger the overexpression of the HXK signaling pathway resulting on promoting cell division (for a recent review see [34]). Thus, it is possible that low sugar levels promote apospory by retarding senescence and high sugar promotes regeneration by promoting cell division.

Ethylene plays an important role in many develop- mental processes in higher plants and ethylene synthesis is regulated not only by developmental cues but also by other hormones namely auxin, gibberellins and cytokinin and is significantly enhanced by stresses such as wounding, drought, cold or pathogen and insect attack [35]. Although the ethylene-signaling pathway in lower plants has not yet been elucidated, it was shown that ethylene plays a positive role in several developmental processes including spore germination [36], gametophyte growth [22], the production of apogamous buds in various ferns [37] and in sporophyte regeneration from fronds and rhizomes [23]. Because glucose at $0.5 \%$ level gave the best result in promoting apospory (Figure 3(a)), this concentration was chosen to determine whether ethylene could further promote this pathway. The initial hint that ethylene might promote apospory came from our observation in which explants produced more gametophytes when the plates were sealed with paraffin compared with those that were not sealed (data not shown). Indeed, the results shown here indicate an enhancing role of ethylene in both regeneration and apospory on detached leaves of C. richardii. Adding ethephon in the media increased not only the number of explants showing regeneration or apospory or both but also the total number of regenerative sporophytes and aposporous gametophytes. Ethephon at high concentration inhibits both processes, which is consistent with previous reports on megasporogenesis in Selaginella [27] and sporophyte regeneration in Platycerium coronarium [23]. Conversely, $5 \mu \mathrm{M}$ STS in the medium reduced regeneration significantly and inhibited apospory completely, presumably by blocking ethylene effects.

The enhancing role of ethylene on apospory is consistent with the known effects of ethylene during early stages of female gametophyte development in land plants. In tobacco, silencing of the gene encoding the ethylene synthesis enzyme, ACC oxidase, or blocking ethylene action by STS, causes ovule development to arrest and megasporocytes abolishment [38]. In Arabidopsis, an ethylene-responsive mutant, constitutive triple response 1 (ctr1), is defective in female gametophyte development [39] and genes encoding ethylene synthesis enzymes are highly expressed during ovule development [40]. In lower plants, spraying Selaginella leaves with $7.6 \mathrm{mg} / \mathrm{L}$ ethephon causes sporangia that would normally produce microspores to produce megaspores instead [27]. Therefore, it is tempting to speculate that ethylene has a comparable role in apospory, which, after all, is the formation of a new gametophyte from a sporophyte, albeit without a spore.

\section{Acknowledgements}

We thank Jonas Baltrusaitis for helping with the SEM. 
Linh T Bui is supported by a fellowship from the Vietnam Overseas Scholarship Program and Avis Cone graduate summer fellowships. Amelia Hurst is supported by fellowships from the University of Iowa Biology Honors Program and the ICRW Fellowship Program.

\section{REFERENCES}

[1] W. N. Steil, “Apogamy, Apospory, and Parthenogenesis in the Pteridophytes," Botanical Review, Vol. 5, No. 8, 1939, pp. 433-453. doi:10.1007/BF02878704

[2] P. R. Bell, "Apospory and Apogamy: Implications for Understanding the Plant Life Cycle," International Journal of Plant Science, Vol. 153, No. 3 (Part 2: The Katherine Esau International Symposium), 1992, pp. S123S136.

[3] T. G. Walker, "The Cytogenetics of Ferns," In: A. F. Dyer, Ed., The Experimental Biology of Ferns, Academic Press, London, 1979, pp. 87-132.

[4] V. Raghavan, "Developmental Biology of Fern Gametophytes," Cambridge University Press, Cambridge, 1989. doi:10.1017/CBO9780511529757

[5] A. R. Cordle, E. E. Irish and C.-L. Cheng, "Apogamy Induction in Ceratopteris richardii," International Journal of Plant Science, Vol. 168, No. 4, 2007, pp. 361-369. doi:10.1086/511049

[6] M. H. Munroe and I. M. Sussex, "Gametophyte Formation in Bracken Fern Root Cultures," Canadian Journal of Botany, Vol. 47, No. 4, 1969, pp. 617-621. doi: $10.1139 / \mathrm{b} 69-088$

[7] B. DeYoung, T. Weber, T. Hass and J. A. Banks, "Generating Autotetraploid Sporophytes and Their Use in Analyzing Mutations Affecting Gametophyte Development in the Fern Ceratopteris," Genetics, Vol. 147, No. 2, 1997, pp. 809-814.

[8] A. R. Cordle, L. T. Bui, E. E. Irish and C.-L. Cheng, "Laboratory-Induced Apogamy and Apospory in Ceratopteris rihardii," In: H. Fernandes, A. Kumar and M. A. Revilla, Eds., Working with Ferns, Springer, New York, 2011, pp. 25-36. doi:10.1007/978-1-4419-7162-3_3

[9] V. Menéndez, R. Arbesú, M. Somer, A. Revilla and H. Fernández, "From Spore to Sporophyte: How to Proceed in Vitro," In: H. Fernandes, A. Kumar and M. A. Revilla, Eds., Working with Ferns, Springer, New York, 2011, pp. 97-110.

[10] B. J. W. Dekkers, J. A. M. J. Schuurmans and S. C. M. Smeekens, "Interaction between Sugar and Abscisic Acid Signalling during Early Seedling Development in Arabidopsis," Plant Molecular Biology, Vol. 67, No. 1-2, 2008, pp. 151-167. doi:10.1007/s11103-008-9308-6

[11] M. Ramon, F. Rolland and J. Sheen, "Sugar Sensing and Signaling," The Arabidopsis Book (TAB), Vol. 6, 2008, pp. $1-22$. doi: $10.1199 /$ tab.0117

[12] J. Hanson and S. C. M. Smeekens, "Sugar Perception and Signaling-An Update," Current Opinions in Plant Biology, Vol. 12, No. 5, 2009, pp. 562-567. doi:10.1016/j.pbi.2009.07.014

[13] A. Nilsson, T. Olsson, M. Ulfstedt, M. Thelander and H. Ronne, "Two Novel Types of Hexokinases in the Moss Physcomitrella patens," BMC Plant Biology, Vol. 11, 2011, p. 32. doi:10.1186/1471-2229-11-32

[14] T. Olsson, M. Thelander and H. Ronne, "A Novel Type of Chloroplast Stromal Hexokinase Is the Major Glucose Phosphorylating Enzyme in the Moss Physcomitrella patens," Journal of Biological Chemistry, Vol. 278, 2003, pp. 44439-44447. doi:10.1074/jbc.M306265200

[15] H. Weber, L. Borisjuk and U. Wobus, "Sugar Import and Metabolism during Seed Development," Trends in Plant Science, Vol. 2, No. 5, 1997, pp. 169-174. doi:10.1016/S1360-1385(97)85222-3

[16] L. Zhou, J. C. Jang, T. L. Jones and J. Sheen, "Glucose and Ethylene Signal Transduction Crosstalk Revealed by an Arabidopsis Glucose Insensitive Mutant," PNAS, Vol. 95, No. 17, 1998, pp. 10294-10299. doi:10.1073/pnas.95.17.10294

[17] S. I. Gibson, R. J. Laby and D. G. Kim, "The Sugar-Insensitive1 (sis1) Mutant of Arabidopsis Is Allelic to ctr1," Biochemistry and Biophysics Reserach Communication, Vol. 280, No. 1, 2001, pp. 196-203. doi:10.1006/bbrc.2000.4062

[18] S. Gazzarrini and P. McCourt, "Genetic Interactions between ABA, Ethylene and Sugar Signaling Pathways," Current Opinion in Plant Biololgy, Vol. 4, No. 5, 2001, pp. 387-391. doi:10.1016/S1369-5266(00)00190-4

[19] S. Gazzarrini and P. McCourt, "Cross-Talk in Plant Hormone Signaling: What Arabidopsis Mutants Are Telling Us," Annuals of Botany, Vol. 91, No. 6, 2003, pp. 605612. doi:10.1093/aob/mcg064

[20] A. L. Eveland and D. P. Jackson, "Sugars, Signaling, and Plant Development," Journal of Experimental Botany, 2011.

[21] Y.-H. Cho, J. Sheen and S.-D. Yoo, "Low Glucose Uncouples Hexokinase1-Dependent Sugar Signaling from Stress and Defense Hormone Abscisic Acid and $\mathrm{C}_{2} \mathrm{H}_{4}$ Responses in Arabidopsis," Plant Physiology, Vol. 152, No. 3, 2010, pp. 1180-1182. doi:10.1104/pp.109.148957

[22] P. M. Miller, H. C. Sweet and J. H. Miller, "Growth Regulation by Ethylene in Fern Gametophytes. I. Effects on Protonemal and Rhizoidal Growth and Interaction with Auxin," American Journal of Botany, Vol. 57, No. 2, 1970, pp. 212-217. doi:10.2307/2440515

[23] S. H. Kwa, Y. C. Wee and P. P. Kumar, "Role of Ethylene in the Production of Sporophytes from Platycerium coronarium (Koenig) Desv. Frond and Rhizome Pieces Cultured in Vitro," Journal of Plant Growth Regulation, Vol. 14, No. 4, 1995, pp.183-189. doi:10.1007/BF00204910

[24] D. J. Osborne, J. Walters, B. V. Milborrow, A. Norville and L. M. C. Stange, "Special Publication Evidence for a Non-ACC Ethylene Biosynthesis Pathway in Lower Plants," Phytochemistry, Vol. 42, No. 1, 1996, pp. 51-60. doi:10.1016/0031-9422(96)00032-5

[25] J. Chernys and H. Kende, "Ethylene Biosynthesis in 
Regnellidium diphyllum and Marsilea quadrifolia," Planta, Vol. 200, 1996, pp. 113-118. doi:10.1007/BF00196657

[26] A. Anterola and E. Shanle, "Genomic Insights in Moss Gibberellins Biosynthesis," The Bryologist, Vol. 111, No. 2, 2008, pp. 218-230. doi:10.1639/0007-2745(2008)111[218:GIIMGB]2.0.CO; $\underline{2}$

[27] K. E. Brooks, "Reproductive Biology of Selaginella. I. Determination of Megasporangia by 2-Chloroethylphosphonic Acid, an Ethylene Releasing Compound," Plant Physiology, Vol. 51, No. 4, 1973, pp. 718-722. doi:10.1104/pp.51.4.718

[28] S. F. Yang, "Ethylene Evolution from 2-Chloroethylphosphonic Acid," Plant Physiology, Vol. 44, No. 8, 1969, pp. 1203-1204. doi:10.1104/pp.44.8.1203

[29] F. B. Abeles, P. W. Morgan and M. E. Saltveit Jr., "Ethylene in Plant Biology," 2nd Edition, Academic Press, Inc., San Diego, 1992, pp. 1-13.

[30] L. G. Hickok, T. R. Warne and R. S. Fribourg, "The Biology of the Fern Ceratopteris and Its Use as a Model System," Inernational Journal of Plant Science, Vol. 156, No. 3, 1995, pp. 332-345. doi:10.1086/297255

[31] W. C. Yang and V. Sundaresan, "Genetics of Gametophyte Biogenesis in Arabidopsis," Current Opinion in Plant Biology, Vol. 3, No. 1, 2000, pp. 53-57. doi:10.1016/S1369-5266(99)00037-0

[32] A. M. Hirsch, "The Effect of Sucrose on the Differentiation of Excised Fern Leaf Tissue into Either Gametophytes or Sporophytes," Plant Physiology, Vol. 56, No. 3, 1975, pp. 390-393. doi:10.1104/pp.56.3.390

[33] J. Ambrožič-Dolinšek, M. Camloh, B. Bohanec and J. Žel,
"Apospory in Leaf Culture of Staghorn Fern Platycerium bifurcatum," Plant Cell Reports, Vol. 20, No. 9, 2002, pp. 791-796. doi:10.1007/s00299-001-0403-2

[34] M. R. Bolouri-Moghaddam, K. L. Roy, L. Xiang, F. Rolland and W. Van den Ende, "Sugar Signaling and Antioxidant Network Connections in Plant Cells," FEBS Journal, Vol. 277, No. 9, 2010, pp. 2022-2037. doi:10.1111/j.1742-4658.2010.07633.x

[35] S. D. Yoo, Y. Cho and J. Sheen, "Emerging Connections in the Ethylene Signaling Network," Cell Press, Elsevier Ltd., 2009.

[36] T. R. Warner and L. G. Hickok, “(2-Chloroethyl) Phosphonic Acid Promotes Germination of Immature Spores of Ceratopteris richardii Brongn," Plant Physiology, Vol. 83, No. 4, 1987, pp. 723-725. doi:10.1104/pp.83.4.723

[37] H. W. Elmore and D. P. Whittier, "The Role of Ethylene in the Induction of Apogamous Buds in Pteridium Gametophytes," Planta, Vol. 111, No. 1, 1973, pp. 85-90. doi:10.1007/BF00386738

[38] D. De Martinis and C. Mariani, "Silencing Gene Expression of the Ethylene-Forming Enzyme Results in a Reversible Inhibition of Ovule Development in Transgenic Tobacco Plants," The Plant Cell, Vol. 11, No. 6, 1999, pp. 1061-1071. doi:10.1105/tpc.11.6.1061

[39] G. N. Drews, D. Lee and C. A. Christensen, "Genetic Analysis of Female Gametophyte Development and Function," The Plant Cell, Vol. 10, 1998, pp. 5-17.

[40] P. Carbonell-Bejerano, C. Urbez, A. Granell, J. Carbonell and M. Perez-Amador, "Ethylene Is Involved in Pistil Fate by Modulating the Onset of Ovule Senescence and the GA-Mediated Fruit Set in Arabidopsis," BMC Plant Biology, Vol. 11, No. 84, 2011, pp. 11-84. 